\title{
MicroRNA-1 and microRNA-499 downregulate the expression of the ets1 proto-oncogene in HepG2 cells
}

\author{
WEI WEI ${ }^{1,2}$, ZHENG HU $^{1}$, HANJING FU $^{1}$, YI TIE ${ }^{1}$, HAIHONG ZHANG $^{2}$, YONGGE WU $^{2}$ and XIAOFEI ZHENG ${ }^{1}$ \\ ${ }^{1}$ Beijing Institute of Radiation Medicine, Beijing; ${ }^{2}$ National Engineering Laboratory for \\ AIDS Vaccine, College of Life Science, Jilin University, Changchun, P.R. China
}

Received March 21, 2012; Accepted May 14, 2012

DOI: $10.3892 /$ or.2012.1850

\begin{abstract}
MicroRNAs may function to promote or suppress tumor development, depending on the cellular context. The important role of microRNAs in regulating molecular pathways underlying tumorigenesis has been emphasized in hepatocellular carcinoma (HCC). MicroRNAs regulate gene expression via post-transcriptional mechanisms by inhibiting translation or by degrading mRNA. In this study, we show that microRNA-1 (miR-1) and microRNA-499 (miR-499) are capable of repressing the expression of the ets 1 protooncogene, which plays a fundamental role in the extracellular matrix (ECM) degradation, a process required for tumor cell invasion and migration. We used luciferase reporter assays to demonstrate that $m i R-1$ and $m i R-499$ target the 3 ' untranslated region (UTR) of ets 1 . Overexpression of $m i R-1$ and $m i R-499$ in HepG2 cells led to downregulation of ets 1 mRNA and protein as assessed by quantitative reverse transcription PCR and western blot analysis. Furthermore, overexpression of $m i R-1$ and $m i R-499$ inhibited the invasion and migration of HepG 2 cells in matrigel invasion and transwell migration assays, respectively. These results suggest that $m i R-1$ and $m i R-499$ may play an important role in the pathogenesis of $\mathrm{HCC}$ by regulating etsl.
\end{abstract}

\section{Introduction}

Hepatocellular carcinoma (HCC) is the sixth most common malignancy and the third most common cause of cancer-related deaths worldwide, claiming over one million lives annually (1). The highest incidence rates are reported in East Asia (2). The

Correspondence to: Professor Xiaofei Zheng, Beijing Institute of Radiation Medicine, 27 Taiping Road, Beijing 100850, P.R. China E-mail: xfzheng100@126.com

Professor Yongge Wu, National Engineering Laboratory for AIDS Vaccine, College of Life Science, Jilin University, Changchun 130012, P.R. China

E-mail:ygwu@jlu.edu.cn

Key words: microRNA, microRNA-1, microRNA-499, ets1, invasion, migration, HepG2 cell prognosis of patients with $\mathrm{HCC}$ is poor, with a 5-year survival rate after diagnosis of $\sim 10 \%$ (3).

Ets1 is the founding member of the Ets family of transcription factors and has been shown to promote invasive behavior in multiple cell types (4-10). The regulation of matrix metalloproteases MMP-1, MMP-3, MMP-9 and urokinase type plasminogen activator ( $\mathrm{uPA}$ ) expression have been ascribed to Ets1 (4-10). Expression of Ets1 is also associated with poor prognosis in patients with tumors including breast cancer, ovarian tumor, and hepatocellular carcinoma.

Various molecular alterations occur in pre-neoplastic nodules and escalate in HCC, including dysregulation of well-known molecular pathways in carcinogenesis (11-14). The important role of microRNAs (miRNAs) in regulating these pathways has been emphasized. MiRNAs are small (18-24 nucleotides), evolutionarily conserved, endogenous, single-stranded, non-coding RNA molecules, that negatively modulate gene expression in animals and plants. Mature miRNAs operate via sequence-specific interactions with the 3' untranslated region (UTR) of cognate mRNA targets, causing suppression of translation and mRNA decay $(15,16)$. A large body of evidence suggests that the multigene regulatory capacity of miRNAs is dysregulated and exploited in cancer. Indeed, miRNA loci are often targeted by genetic and epigenetic defects, and miRNA signatures facilitating tumor classification and the prediction of clinical outcome have been reported (17,18). A global reduction of miRNA abundance appears to be a general trait of human cancers, playing a causal role in the transformed phenotype (19-21). Aberrant expression of miRNA has also been linked to a variety of cancers, including HCC (22-25).

In the current study, we show that the etsl proto-oncogene, which is highly expressed in HCC (26), is targeted by $m i R-1$ and $m i R-499 . M i R-1$ and $m i R-499$ specifically inhibit the expression of Ets1. Overexpression of $m i R-1$ and $m i R-499$ in the HepG2 HCC cell line inhibited cellular invasion and migration. Taken together, these results suggest that Est 1 is negatively regulated by $m i R-1$ and $m i R-499$ in HepG2 cells, which may contribute to the invasive and migratory potential of hepatocellular carcinoma.

\section{Materials and methods}

Cell Culture. HepG2 and HEK 293 cell lines (American Type Culture Culture Collection, Manassas, VA, USA) were 
maintained in Dulbecco's modified Eagle medium (DMEM) (Gibco-BRL, Grand Island, NY, USA) containing 10\% (v/v) fetal bovine serum (FBS) supplemented with $100 \mathrm{U} / \mathrm{ml}$ penicillin and $100 \mu \mathrm{g} / \mathrm{ml}$ streptomycin, at $37^{\circ} \mathrm{C}$ with $5 \% \mathrm{CO}_{2}$.

Vector construction. For construction of miRNA expression plasmids, $m i R-1$ and $m i R-499$ precursors were amplified from human genomic DNA by PCR using the primer pairs: $m i R-1$, F, 5'-TAGAAGCTTGCCTCTGAGCTGCCTTCTCTA-3' and R, 5'-TATCTCGAGCACCACAGCCGCCTGGCTGGC-3'; $m i R-499$, F, 5'-TAGAAGCTTGTGTCCCAGCTGCACA AGGTA-3' and R, 5'-TATCTCGAGTGTCTCCCATCACCA CCACCA-3'. PCR products were cloned into pcDNA3.0 (Invitrogen, Carlsbad, CA, USA). In the same way, the miRNA expression plasmids of $m i R-139, m i R-181 a, m i R-200 b$, $m i R-221, m i R-365$ and $m i R-429$ had been constructed in our laboratory. For the construction of luciferase reporter vectors, 3'UTR segments of etsl (Ets1-3'UTR-1 and Ets1-3'UTR-2) were amplified from human genomic DNA using the primer pairs: Ets1-3'UTR-1, F, 5'-ACGTCTAGACTGTGAGTATA ACTCCTGCAG-3' and R, 5'-GATCATATGATATGAAA TCAGGCTACAGTA-3'; Ets1-3'UTR-2, F, 5'-ACGTCTAG AGCAAGTGACATTGTCACATCA-3' and R, 5'-GATCATA TGCACCAATCAGAAAGCCGTACA-3. Mutant inserts containing substitutions in the miRNA complementary sites were generated by PCR using the primers: Ets1-3'UTR-1mut, F, 5'-TTGTTGAACTCTTACCTCGCCGGGCAAGAATTT CAAGGAACC-3' and R, 5'-GGTTCCTTGAAACTTCTT GCCCGGCGAGGTAAGAGTTCAACAA-3'; Ets1-3'UTR2mut, F, 5'-TTTTTTTCTTAAAAATCCGGCCGGGCTCTA AGGTGGTCTCAG-3' and R, 5'-CTGAGACCACCTTAGAG CCCGGCCGGATTTTTAAGAAAAAAA-3'. PCR products were cloned into the modified pGL3 control vector (Promega, Madison, WI, USA) immediately downstream of the stop codon of the luciferase gene. Wild-type and mutant inserts were confirmed by sequencing.

miRNAs, small interfering RNA (siRNA) and transfection. The $m i R-1$ and $m i R-499$ duplexes, ets 1 and negative control siRNAs were designed and synthesized by GenePharma (Shanghai, China). The sequences are as follows (sense/antisense): $m i R-1,5^{\prime}$-UGGAAUGUAAAGAAGUAUGUAU-3'/ 5'-AUACAUACUUCUUUACAUUCCA-3'; miR-499, 5'-UUA AGACUUGCAGUGAUGUUU-3'/5'-AAACAUCACUGCAA GUCUUAA-3'; ets 1 siRNA, 5'-ACUUGCUACCAUCCCGU ACTT-3'/5'-GUACGGGAUGGUAGCAAGUTT-3'; negative control siRNA, 5'-UUCUCCGAACGUGUCACGUTT -3'5'-ACGUGACACGUUCGGAGAATT-3'. Transfection was performed using Lipofectamine 2000 (Invitrogen). In brief, cells were seeded in six-well plates to reach an optimum density of $50 \%$ confluency after $24 \mathrm{~h}$. For transfections, siRNA $(20 \mu \mathrm{M})$ or miRNA $(20 \mu \mathrm{M})$ was combined with $5 \mu \mathrm{l}$ of Lipofectamine 2000 and $250 \mu \mathrm{l}$ of Opti-MEM medium (Gibco-BRL). This mixture was added to cells and incubated for $6 \mathrm{~h}$ before replacing with fresh medium. Total-RNA and protein were extracted $48 \mathrm{~h}$ after transfection for use in qRT-PCR and western blot analysis.

Luciferase reporter assays. HEK 293 cells were plated in 24 -well plates to reach $80-90 \%$ confluency. Cells were co-transfected with luciferase reporter vectors (100 ng) containing the ets 1 3'UTR (pGL3m-Ets 1-3'UTR-1 and pGL3mEts1-3'UTR-2) or etsl 3'UTR mutant (pGL3-Ets1-3'UTR-1mut and pGL3-Ets1-3'UTRmut-2mut) and pRL-TK control Renilla luciferase vector (Promega) (8 ng) using Lipofectamine 2000 (Invitrogen). Luciferase activity was measured by Dual luciferase assays (Promega) $48 \mathrm{~h}$ after transfection.

$R N A$ extraction and $q R T-P C R$. Total-RNA was extracted with TRIzol reagent (Invitrogen) according to the manufacturer's instructions. cDNA was synthesized using oligo(dt) primers and Impro-II reverse transcriptase (Promega) according to the manufacturer's instructions. qRT-PCR reactions were prepared using SYBR Premix Ex Taq (Takara, Kyoto, Japan). Reactions were performed in triplicate using an Mx3000P real-time PCR instrument (Agilent Technologies, Santa Clara, CA, USA). The PCR primers were: etsl, F, 5'-TGGAGTC AACCCAGCCTATC-3' and R, 5'-TCTGCAAGGTGTCTGTC TGG-3'; GAPDH, F, 5'-TCAGTGGTGGACCTGACCTG-3' and R, 5'-TGCTGTAGCCAAATTCGTTG-3'. Expression of etsl was calculated according to the delta-delta $\mathrm{Ct}$ method, normalizing to GAPDH.

Western blot analysis. Total cell lysates were extracted using sodium dodecyl sulfate (SDS) buffer. Proteins were resolved by $12 \%$ SDS-PAGE and transferred onto polyvinylidene fluoride membranes. Membranes were probed with monoclonal antibodies to Ets1 (sc-55581; Santa Cruz Biotechnology, Inc., Santa Cruz, CA, USA) and $\beta$-actin (sc-47778; Santa Cruz Biotechnology). Detection was performed with Supersignal (Pierce, Rockford, IL, USA) chemiluminescence reagent. Quantitative analysis was performed using Quantity One software (Bio-Rad, Hercules, CA, USA).

Transwell cell invasion and migration assays. For invasion assays, transfected HepG2 cells were serum-starved for $18 \mathrm{~h}$ in DMEM containing 0.1\% (v/v) FBS. Cells were trypsinized and resuspended in the same medium and $2 \times 10^{5}$ cells were added to the upper chamber of each well $(6.5 \mathrm{~mm}$ in diameter, $8 \mu \mathrm{m}$ pore size; Corning, Inc., Corning, NY, USA) coated with $30 \mathrm{mg} / \mathrm{cm}^{2}$ matrigel extracellular matrix (ECM) gel (SigmaAldrich, St. Louis, MO, USA). Medium containing $0.1 \%(\mathrm{v} / \mathrm{v})$ FBS, supplemented with hepatocyte growth factor (HGF) $(20 \mathrm{ng} / \mathrm{ml})$ (ProSpec-Tany TechnoGene, Ltd., East Brunswick, NJ, USA) was placed in the lower compartment of the chamber. After incubation for $24 \mathrm{~h}$ at $37^{\circ} \mathrm{C}$, cells on the upper membrane surface were removed by carefully wiping with a cotton swab, and the filters were fixed by treatment with $95 \%(\mathrm{v} / \mathrm{v})$ ethanol for $30 \mathrm{~min}$. Cells were stained with $0.2 \%(\mathrm{w} / \mathrm{v})$ crystal violet solution for $30 \mathrm{~min}$. Cells adhering to the undersurface of the filter were counted (five high-power fields/chamber) using an inverted microscope. Migration assays were performed as described above, excluding the use of matrigel and with an incubation time of $12 \mathrm{~h}$.

Statistical analysis. All values are reported as the means \pm standard deviation. Differences were assessed by two-tailed Student's t-test of Excel software. $\mathrm{P}<0.05$ was considered statistically significant. 
A

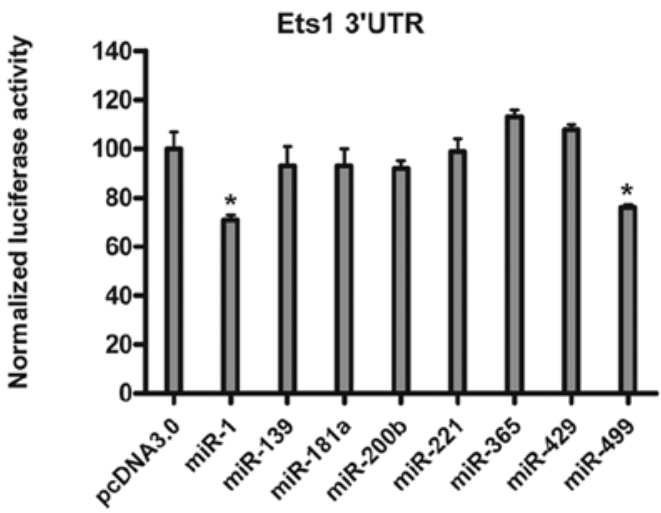

B

Ets1 3' UTR

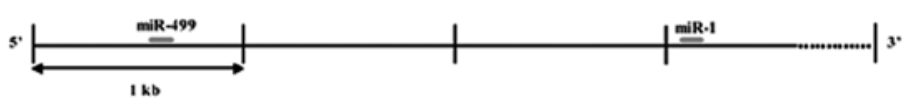

Target site of miR-499

H. Sapiens GCCAGUAUUUUUU-UU----CUUAAAA--------AUCGUCUUAAGCUCUAAGGU M. Musculus GCCAGUAUUUUUU-UUUCCUUAAAAAAAU--AUCGUCUUAAGCUCUAAGGU R. Norvegicus GCCAGUAUUUUUU-UU-...-CUUAAAAAAA-.-AUCGUCUUAAGCUCUAAGGU C. Familiaris GCCAGUAUUUUUU-UU.....CUUAAAA-........-AUCGUCUUAAGCUCUAAGGU

$\mathrm{C}$

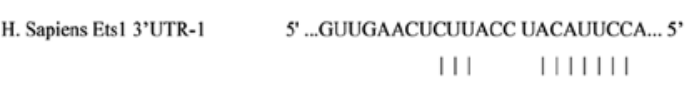
H. Sapiens Ets1 3'UTR -2

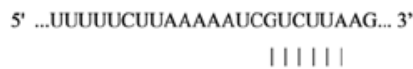
miR-1 3' UauguaugahgaAauguaggu 3' $\operatorname{miR}-499$ 3' UUUguagugacguncagaauU 5 ,

H. Sapiens Ets1 3'UTR mutant-1 5 '...GUUGAACUCUUACC UCGCCGGGC... 5 , | | | miR-1 3. UauguaugahaaAuguaggu 5 ,

H. Sapiens Ets1 3'UTR mutant-2 5' ...UUUUUCUUAAAAAUCCGGCCGGG... 3' $\operatorname{miR}-499$ 3. UUUguagugacguUcagaAuU 5,

D

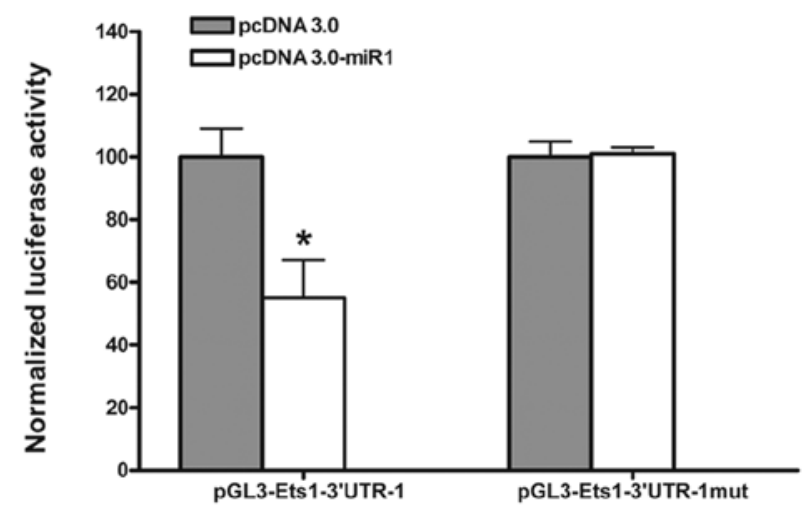

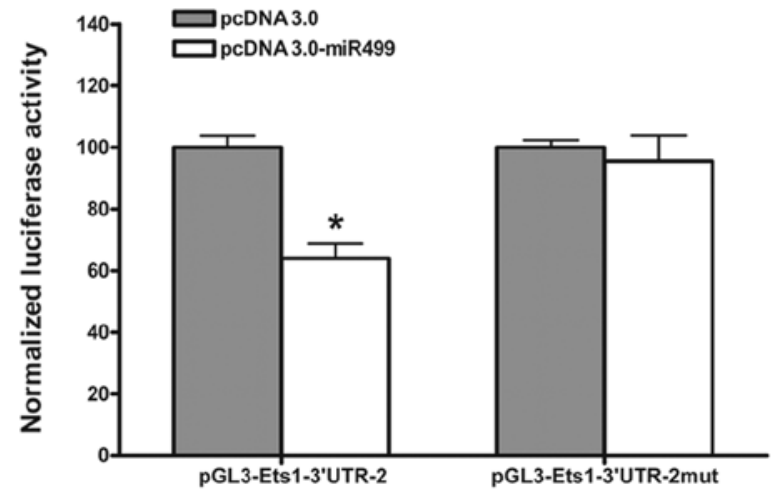

Figure 1. $m i R-1$ and $m i R-499$ target the 3'UTR of $e t s 1 m R N A$. (A) Screening putative $e t s 1$ miRNAs by luciferase reporter assays. To identify miRNAs capable of regulating the expression of ets1 mRNA, the human ets1 3'UTR was subcloned into the pGL3m vector. HEK 293 cells were co-transfected with luciferase-Ets1 plasmid and pcDNA3.0 control plasmid or miRNA-expressing plasmids. Luciferase activity was normalized to Renilla luciferase expression. Results represent the mean of biological duplicate assays \pm the standard deviation $\left({ }^{*} \mathrm{P}<0.05\right)$. (B) Upper panel, schematic representation of the ets 13 'UTR. Gray bars indicate predicted $m i R-1$ and $m i R-499$ target sites. Lower panel, the target site of $m i R-1$ and $m i R-499$ in the $e t s 1$ 3'UTR is conserved among mammalian species (shown in gray). (C) Predicted duplex formation between $m i R-1$ and $m i R-499$ and the targeted ets 1 3'UTR-1 or ets 1 3'UTR-2). The ets 1 3'UTR-1mut or 3'UTR-2mut sequences are identical to wild-type sequences, except for a 7 or 8 bp substitution (light gray letters). (D) pGL3m-Ets1-3'UTR-1 or pGL3m-Ets1-3'UTR-2 reporter plasmids were co-transfected into HEK 293 cells with pcDNA3.0 (gray columns), pcDNA3.0-miR-1 or pcDNA3.0-miR-499 (white columns). Luciferase activity was normalized to Renilla luciferase expression. Results represent the mean of biological triplicate assays \pm the standard deviation ("P<0.05).

\section{Results}

Interaction of miR-1 or miR-499 with the 3'UTR of Ets1 $m R N A$. In order to identify miRNAs regulating Ets1, we used TargetScan (www.targetscan.org), an online software program, to predict miRNAs targeting 3'UTR of the ets 1 mRNA. This analysis revealed that the 3'UTR of ets 1 contains putative sites for $>20$ miRNAs. We tested the ability of a subset of these miRNAs (miR-1, miR-139, miR-181a, miR-200b, miR-221, $m i R-365, m i R-429$ and $m i R-499)$ to target the ets 1 3'UTR 
A

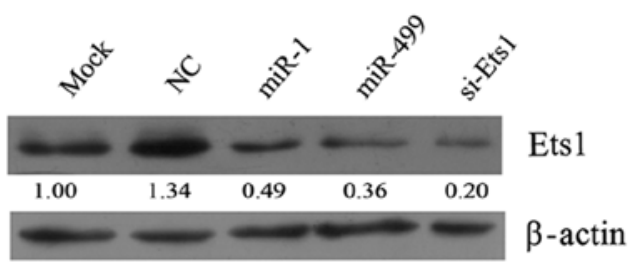

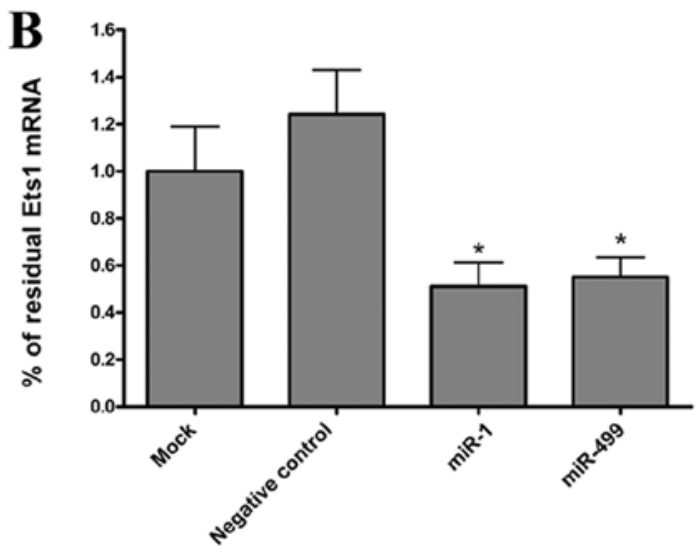

Figure 2. $m i R-1$ and $m i R-499$ regulate Ets1 expression at the post-transcriptional level. (A) Ets1 protein was measured in HepG2 cells by western blot analysis $48 \mathrm{~h}$ after transfection with synthetic $m i R-1$ and $m i R-499$ duplexes, or Ets1 siRNA. $\beta$-actin was used as an internal loading control. (B) The effect of $m i R-1$ and $m i R-499$ on ets 1 mRNA expression was analyzed by qRT-PCR. Ets1 levels were calculated using GAPDH as an internal control. Results represent the mean of triplicate qRT-PCR assays \pm standard deviation $(" \mathrm{P}<0.05)$.
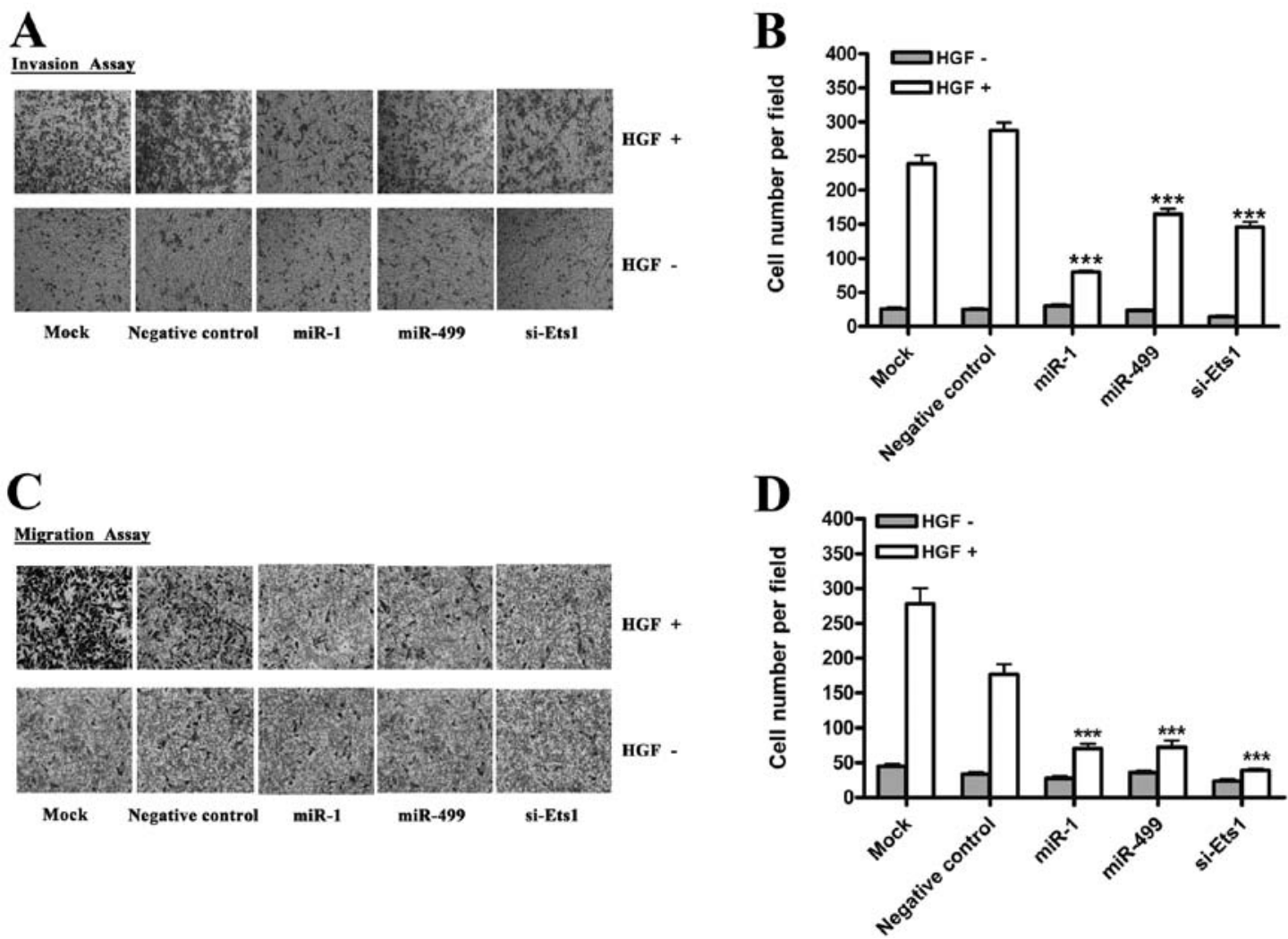

Figure 3. $m i R-1$ and $m i R-499$ suppress invasion and migration of HepG2 cells by inhibiting Ets1 expression. (A) Cell invasion was assayed using matrigel invasion chambers. HepG2 cells were transfected with $m i R-1$ and $m i R-499$ duplexes or Ets1 siRNA and plated $48 \mathrm{~h}$ after transfection in 24 -well transwell plates. Mock-transfected cells (no miRNA or siRNA) or cells transfected with negative control siRNA were used as controls. Cells were added to the upper chamber of each well. After $24 \mathrm{~h}$ of incubation, cells that invaded through the pores to the under surface of the membrane were fixed, stained and counted. Five random microscopic fields were counted for each treatment. (B) Cell numbers represent the average count of five random microscopic fields. Each bar represents the mean \pm standard deviation of the counts from a single representative experiment $\left({ }^{* * *} \mathrm{P}<0.001\right)$. (C) $m i R-1$ and $m i R-499$ blocked cell migration as assayed in transwell chambers, following incubation for $12 \mathrm{~h}$ and using HGF $(20 \mathrm{ng} / \mathrm{ml})$ as a chemoattractant. Cells that migrated through the filter were fixed and stained with crystal violet. Representative images of the lower surface of membrane are shown. (D) Results represent the average of three experiments \pm standard deviation from a representative experiment $\left({ }^{* * *} \mathrm{P}<0.001\right)$.

using luciferase reporter assays. Our analysis showed that only $m i R-1$ and $m i R-499$ induced an obvious decrease in relative luciferase activity (Fig. 1A). Further investigation showed that the putative target sites for $m i R-1$ and $m i R-499$ are conserved in mammalian species (Fig. 1B). The target sites for $m i R-499$ and $m i R-1$ locate in the front and rear fragment of 3'UTR of ets 1 mRNA, respectively. To investigate this potential interaction experimentally, the 3'UTR of ets 1 mRNA was divided into two fragments, Ets1-3'UTR-1 and Ets1-3'UTR-2, and sub-cloned into a modified pGL3 control plasmid (pGL3m), as 


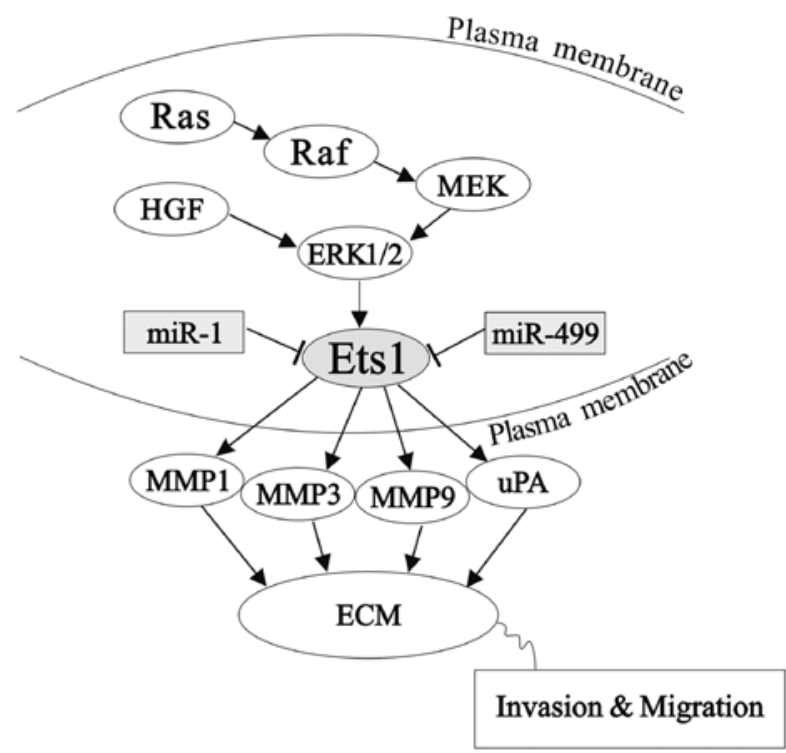

Figure 4. A schematic representation showing how miR-1 and $m i R-499$ modulate the invasive and migratory behaviors of HepG2 cells via modulation of Ets1.

previously described (27). We then tested the ability of $m i R-I$ or $m i R-499$ to inhibit luciferase activity of pGL3m-Ets13'UTR-1 or pGL3m-Ets1-3'UTR-2 following co-transfection into HEK 293 cells. Our analysis demonstrated that both $m i R-1$ and $m i R-499$ induced a significant decrease in relative luciferase activity ( $\sim 40 \%)$ compared with vector transfected cells (Fig. 1D). To test the specificity of this interaction, $m i R-1$ and $m i R-499$ overexpression constructs were co-transfected with ets 1 luciferase reporter constructs containing substitutions disrupting the miRNA target sites (Fig. 1C). We did not observe any decrease in relative luciferase activity in miRNA transfected cells compared with vector control (Fig. 1D).

miR-1 and miR-499 downregulate Ets1 expression. To investigate whether $m i R-1$ or $m i R-499$ affect endogenous Ets 1 protein expression, we transfected $m i R-1$ and $m i R-499$ duplexes into HepG2 cells and analyzed Ets1 expression after $48 \mathrm{~h}$ by western blot analysis. As a positive control, cells were also transfected with Ets1 siRNA. We found that $m i R-1$ and $m i R-499$ dramatically reduced the expression of Etsl protein compared to negative control (Fig. 2A). qRT-PCR analysis of ets 1 mRNA expression $48 \mathrm{~h}$ after transfection with $m i R-1$ and $m i R-499$ duplexes, revealed that the level of Ets1 mRNA was also significantly reduced compared to negative control (Fig. 2B). There were no significant differences on Ets1 protein and mRNA levels between overexpression of individual miRNAs or co-transfection of both $m i R-1$ and $m i R-499$.

miR-1 and miR-499 negatively regulate cell invasion and migration in vitro. HGF (hepatocyte growth factor), a cytokine also known as scatter factor (SF), can significantly promote the invasion of hepatoma carcinoma cells (28). Moreover, Ets1 has been shown to play a key role in the acquisition of invasive behavior by inducing the expression of $M M P-1, M M P-3$, $M M P-9$ and $U P A$ (4). Given that $m i R-1$ and $m i R-499$ are capable of affecting Ets1 expression, we examined the effect of overexpressing these miRNAs on HGF-induced invasiveness of HepG2 cells using the matrigel invasion assay system. As shown in Fig. 3A, $m i R-1$ and $m i R-499$ significantly reduced HGF-induced invasion of HepG2 cells. Next, we examined the effect of $m i R-1$ and $m i R-499$ on HGF-induced migration of HepG2 cells using transwell migration assays. Similar to the invasion assays, $m i R-1$ and $m i R-499$ also inhibited the migration behavior of HepG2 cells (Fig. 3C). To eliminate the possibility of off-target effects, we transfected cells with Ets1 siRNA. In a similar manner to miRNA overexpression, we found that knockdown of Ets1 inhibited the invasion and migration induced by HGF (Fig. 3A and C).

\section{Discussion}

Growing evidence indicates that Ets1 plays a key role in the invasive behavior of many mammalian tumors. In this study, we demonstrate that $m i R-1$ and $m i R-499$ negatively regulate the ets 1 proto-oncogene at the post-transcriptional level, via conserved sites within the 3'UTR. Furthermore, overexpression of $m i R-1$ or $m i R-499$ inhibited the invasion and migration of HepG2 cells in vitro, emphasizing the essential role of these two miRNAs in hepatic oncogenesis and tumor behavior.

In general, miRNAs may function as both tumor suppressors and oncogenes in tumors. The correlation between the expression of specific miRNAs and cancer has been widely observed. It has also been shown that a global reduction of miRNA abundance may be a general trait of human cancers (19-21). This suggests that miRNAs may have a crucial function in cancer progression (29).

Overexpression of Ets1 is highly associated with many types of cancer. Ets1 expression is generally higher in invasive tumors than in benign tumors $(6,9)$, and is indicative of poor prognosis $(7,9,26,30)$. The expression of Ets1 is also correlated with histological differentiation of HCC (26). This suggests that Ets1 is higher in poorly differentiated HCC and may yield relative biological information to HCC.

Ets1 responsive genes include those encoding certain proteases, including the matrix metalloproteases, $M M P-1, M M P-3$, $M M P-9$ and $u P A$ (4). A schematic representation is shown in Fig. 4. These proteases are involved in ECM-degradation, a key event in invasion. Ets1 expression positively correlates with MMP-1 in angiosarcoma of the skin (31), and with MMP-1 and MMP-9 in ovarian carcinoma cells and stromal fibroblasts in breast and ovarian cancer, respectively $(7,9)$. Ets1 expression also correlates with expression of uPA in lung and brain tumors $(6,8,10)$. Targeted knockdown of Ets1 leads to a decrease in the expression of MMP-1 and MMP-9. Correspondingly, overexpression of Ets1 induced the production of MMP-1, MMP-3 and MMP-9 or MMP-1, MMP-9 and uPA, in hepatoma cells and endothelial cells respectively (32-34).

There is growing evidence to show that Ets1 may also be involved in the regulation of c-Met, the receptor for $\mathrm{HGF} / \mathrm{SF}$, which induces migration (35). Furthermore, c-Met may also activate Ets1, as HGF/SF has been shown to stimulate Ets1 activity through the Ras/Raf/MEK1/ERK1/2 pathway in MDCK cells (36).

In our study, we found that $m i R-1$ and $m i R-499$ inhibit HGF-induced invasion and migration in HCC HepG2 cells, by repressing the expression of the ets 1 proto-oncogene. 
These results indicate that $m i R-1$ and $m i R-499$ may represent candidates for anticancer therapy. In conclusion, $m i R-1$ and $m i R-499$ inhibit Ets1 expression by binding to the 3'UTR of the ets $1 \mathrm{mRNA}$, thereby reducing HGF-induced cell invasion and migration.

\section{Acknowledgements}

This study was partially supported by the Chinese State Key Projects for Basic Research (2010CB912801, 2009CB521804), the Chinese National Natural Science Foundation Projects (81072021) and the Beijing Natural Science Foundation Project (7101007).

\section{References}

1. WHO: The global burden of disease 2004 update. World Health Organization. Geneva, (part 2) pp12-13, 2004.

2. Parkin DM, Bray F, Ferlay $\mathbf{J}$ and Pisani P: Estimating the world cancer burden: Globocan 2000. Int J Cancer 94: 153-156, 2001.

3. London WT and McGlynn KA: Liver cancer. In: Cancer Epidemiology and Prevention. Schottenfeld D, Fraumeni JF Jr (eds). 3rd edition. Oxford University Press, New York, pp763-786, 2006.

4. Sementchenko VI and Watson DK: Ets target genes: past, present and future. Oncogene 19: 6533-6548, 2000

5. Naito S, Shimizu S, Matsuu M, et al: Ets-1 upregulates matrix metalloproteinase-1 expression through extracellular matrix adhesion in vascular endothelial cells. Biochem Biophys Res Commun 291: 130-138, 2002.

6. Kitange G, Tsunoda K, Anda T, et al: Immunohistochemical expression of Ets-1 transcription factor and the urokinase-type plasminogen activator is correlated with the malignant and invasive potential in meningiomas. Cancer 89: 2292-2300, 2000.

7. Behrens P, Rothe M, Wellmann A, Krischler J and Wernert N: The Ets-1 transcription factor is up-regulated together with MMP 1 and MMP 9 in the stroma of pre-invasive breast cancer. J Pathol 194: 43-50, 2001

8. Takanami I, Takeuchi K and Karuke M: Expression of ETS-1 is correlated with urokinase-type plasminogen activator and poor prognosis in pulmonary adenocarcinoma. Tumour Biol 22 205-210, 2001

9. Behrens P, Rothe M, Florin A, Wellmann A and Wernert N: Invasive properties of serous human epithelial ovarian tumors are related to Ets-1, MMP-1 and MMP-9 expression. Int J Mol Med 8: 149-154, 2001.

10. Nakada M, Yamashita J, Okada Y and Sato H: Ets-1 positively regulates expression of urokinase-type plasminogen activator (uPA) and invasiveness of astrocytic tumors. J Neuropathol Exp Neurol 58: 329-334, 1999.

11. El-Serag HB and Rudolph KL: Hepatocellular carcinoma: epidemiology and molecular carcinogenesis. Gastroenterology 132: 2557-2576, 2007.

12. Wurmbach E, Chen YB, Khitrov G, et al: Genome-wide molecular profiles of $\mathrm{HCV}$-induced dysplasia and hepatocellular carcinoma. Hepatology 45: 938-947, 2007.

13. Lee JS and Thorgeirsson SS: Comparative and integrative functional genomics of HCC. Oncogene 25: 3801-3809, 2006.

14. Lemmer ER, Friedman SL and Llovet JM: Molecular diagnosis of chronic liver disease and hepatocellular carcinoma: the potential of gene expression profiling. Semin Liver Dis 26: 373-384, 2006.
15. Bartel DP: MicroRNAs: target recognition and regulatory functions. Cell 136: 215-233, 2009.

16. Ambros V: The functions of animal microRNAs. Nature 431: 350-355, 2004.

17. Calin GA and Croce CM: MicroRNA signatures in human cancers. Nat Rev Cancer 6: 857-866, 2006.

18. Ventura A and Jacks T: MicroRNAs and cancer: short RNAs go a long way. Cell 136: 586-591, 2009.

19. Kumar MS, Lu J, Mercer KL, Golub TR and Jacks T: Impaired microRNA processing enhances cellular transformation and tumorigenesis. Nat Genet 39: 673-677, 2007.

20. Lu J, Getz G, Miska EA, et al: MicroRNA expression profiles classify human cancers. Nature 435: 834-838, 2005.

21. Ozen M, Creighton CJ, Ozdemir M and Ittmann M: Widespread deregulation of microRNA expression in human prostate cancer. Oncogene 27: 1788-1793, 2008.

22. Budhu A, Jia HL, Forgues M, et al: Identification of metastasisrelated microRNAs in hepatocellular carcinoma. Hepatology 47: 897-907, 2008

23. Li LM, Hu ZB, Zhou ZX, et al: Serum microRNA profiles serve as novel biomarkers for HBV infection and diagnosis of HBV-positive hepatocarcinoma. Cancer Res 70: 9798-9807, 2010.

24. Zhou J, Yu L, Gao X, et al: Plasma microRNA panel to diagnose hepatitis B virus-related hepatocellular carcinoma. J Clin Oncol 29: 4781-4788, 2011.

25. Ladeiro Y, Couchy G, Balabaud C, et al: MicroRNA profiling in hepatocellular tumors is associated with clinical features and oncogene/tumor suppressor gene mutations. Hepatology 47: 1955-1963, 2008.

26. Kanda K, Nakayama T, Onizuka S, Tomioka $\mathrm{T}$ and Kanematsu T: Expression of the Ets-1 proto-oncogene is linked to cell differentiation of human hepatocellular carcinoma. Hepatogastroenterology 49: 747-751, 2002.

27. Cui J, Fu H, Feng J, Zhu J, Tie Y, Xing R, Wang C and Zheng X: The construction of miRNA expression library for human. Progr Biochem Biophys 34: 389-394, 2007.

28. Nakamura T, Nishizawa T, Hagiya M, et al: Molecular cloning and expression of human hepatocyte growth factor. Nature 342: 440-443, 1989

29. Calin GA, Sevignani C, Dumitru CD, et al: Human microRNA genes are frequently located at fragile sites and genomic regions involved in cancers. Proc Natl Acad Sci USA 101: 2999-3004, 2004.

30. Span PN, Manders P, Heuvel JJ, et al: Expression of the transcription factor Ets-1 is an independent prognostic marker for relapse-free survival in breast cancer. Oncogene 21: 8506-8509, 2002.

31. Naito S, Shimizu K, Nakashima M, et al: Overexpression of Ets-1 transcription factor in angiosarcoma of the skin. Pathol Res Pract 196: 103-109, 2000.

32. Oda N, Abe M and Sato Y: ETS-1 converts endothelial cells to the angiogenic phenotype by inducing the expression of matrix metalloproteinases and integrin beta3. J Cell Physiol 178: 121-132, 1999.

33. Sato Y, Abe M, Tanaka K, et al: Signal transduction and transcriptional regulation of angiogenesis. Adv Exp Med Biol 476: $109-115,2000$

34. Jiang $\mathrm{Y}, \mathrm{Xu} \mathrm{W}, \mathrm{Lu} \mathrm{J}, \mathrm{He} \mathrm{F}$ and Yang X: Invasiveness of hepatocellular carcinoma cell lines: contribution of hepatocyte growth factor, c-met, and transcription factor Ets-1. Biochem Biophys Res Commun 286: 1123-1130, 2001.

35. Tamagnone L and Comoglio PM: Control of invasive growth by hepatocyte growth factor (HGF) and related scatter factors. Cytokine Growth Factor Rev 8: 129-142, 1997.

36. Paumelle R, Tulasne D, Kherrouche Z, et al: Hepatocyte growth factor/scatter factor activates the ETS1 transcription factor by a RAS-RAF-MEK-ERK signaling pathway. Oncogene 21: 2309-2319, 2002. 